\title{
Dănuţ Marcu
}

Note on Turán's graph

Czechoslovak Mathematical Journal, Vol. 43 (1993), No. 3, 499-501

Persistent URL: http://dml.cz/dmlcz/128421

\section{Terms of use:}

(C) Institute of Mathematics AS CR, 1993

Institute of Mathematics of the Czech Academy of Sciences provides access to digitized documents strictly for personal use. Each copy of any part of this document must contain these Terms of use.

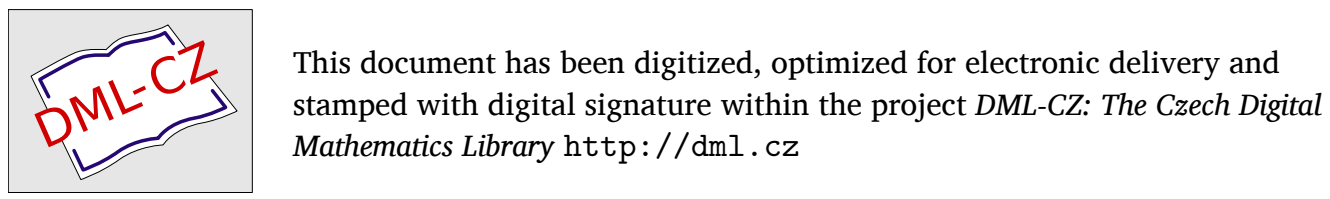




\title{
NOTE ON TURÁN'S GRAPH
}

\author{
DX̃uT MARCU, Bucharest
}

(Received November 11, 1991)

Graphs considered in the paper are finite, undirected and simple (without loops or multiple edges), [1, 2] being followed for terminology and notation. We denote by $S(p, q)$ the Stirling number of the second kind, that is, the number of partitions of a $p$-set into $q$ classes.

A $k$-partite complete graph is a graph consisting of $k$ independent sets, such that two vertices are adjacent if and only if they belong to different independent sets.

Turán's graph, denoted by $T(n, k)$, is a $k$-partite complete graph with $n$ vertices, for which $m$ parts contain $t+1$ vertices and $k-m$ parts contain $t$ vertices, where $n=k t+m$ and $0 \leqslant m \leqslant k-1$. According to [3], $T(n, k)$ is the unique (up to an isomorphism) graph with $n$ vertices which does not contain $(k+1)$-cliques and has the chromatic number equal to $k$, its number of edges being maximal in the class of graphs with these properties.

A $(k+r)$-colouring of a graph with $n$ vertices and the chromatic number equal to $k$ is a partition of its vertex set into $k+r$ classes $(0 \leqslant r \leqslant n-k)$ such that two vertices belonging to the same class are not adjacent, the order of class being indifferent.

Theorem 1. The number $C(n, k, r)$ of $(k+r)$-colourings of $T(n, k)$ is given by

$$
C(n, k, r)=\sum_{\substack{n_{1}, \ldots, n_{k} \geqslant 1 \\ n_{1}+\ldots+n_{k}=k+r}}\left(\prod_{i=1}^{m} S\left(t+1, n_{i}\right)\right) \cdot\left(\prod_{i=m+1}^{k} S\left(t, n_{i}\right)\right) .
$$

Proof. By $n_{i}$ for $i=1, \ldots, k$ let us denote the number of classes of the partition of the $i$-th part of $T(n, k)$ induced by a $(k+r)$-colouring of $T(n, k)$. Then

$$
n_{1}+\ldots+n_{k}=k+r
$$


and

$$
n_{i} \geqslant 1 \quad \text { for } i=1, \ldots, k \text {. }
$$

One can observe that all colourings with $k+r$ classes of $T(n, k)$ are obtained without repetitions from the divisions of $k+r$ into $k$ parts, two divisions being considered different if they differ only by the order of terms.

Obviously, $C(n, k, r)=1$ for $r=0$ and $r=n-k$, and $C(n, k, r)=0$ for $r>n-k$.

Theorem 2. If we denote $[\lambda]_{k}=\lambda(\lambda-1) \ldots(\lambda-k+1)$, then the chromatic polynomial of $T(n, k)$ is equal to

$$
\begin{aligned}
P(T(n, k) ; \lambda)= & \sum_{\substack{p_{1}+\ldots+p_{t+1}=m \\
q_{1}+\ldots+q_{t}=k-m}}\left(\begin{array}{c}
m \\
p_{1}, \ldots, p_{t+1}
\end{array}\right)\left(\begin{array}{c}
k-m \\
q_{1}, \ldots, q_{t}
\end{array}\right) \\
& \times \prod_{i=2}^{t}(S(t+1, i))^{p} \cdot \prod_{j=2}^{t-1}(S(t, j))^{q_{j}}[\lambda]_{p \oplus q}
\end{aligned}
$$

where

$$
p \oplus q=p_{1}+2 p_{2}+\ldots+(t+1) p_{t+1}+q_{1}+2 q_{2}+\ldots+t q_{t}
$$

Pr o of. Obviously, the chromatic polynomial of a graph consisting of $p$ isolated vertices is equal to

$$
\lambda^{p}=\sum_{k=1}^{p} S(p, k)[\lambda]_{k} .
$$

Thus, having in view the method of Read [4], we obtain

$$
P(T(n, k) ; \lambda)=\left(\sum_{p=1}^{t+1} S(t+1, p)[\lambda]_{p}\right)^{m}\left(\sum_{q=1}^{t} S(t, q)[\lambda]_{q}\right)^{k-m}
$$

where, by definition,

$$
[\lambda]_{p}[\lambda]_{q}=[\lambda]_{p+q} \quad \text { for all } p \text { and } q
$$

Using the multinomial formula we obtain the result.

A ck now ledgement. I wish to thank Professor Bohdan Zelinka for his helpful comments, kindness and interest in this paper. 


\section{References}

[1] C. Berge: Graphes et Hypergraphes, Dunod, Paris, 1970.

[2] C. Berge: Principes de Combinatoire, Dunod, Paris, 1968.

[3] P. Turán: On the theory of graphs, Colloq. Math. 3 (1954), 19-30.

[4] R. C. Read: An introduction to chromatic polynomials, J. Combin. Theory 4 (1968), 52-71.

Author's address: Str. Pasului 3, Sect. 2, 70241 Bucharest, Romania. 